MIFPA-13-24

\title{
AdS Dyonic Black Hole and its Thermodynamics
}

\author{
H. Lü ${ }^{1}$, Yi Pang ${ }^{2}$ and C.N. Pope ${ }^{2,3}$ \\ ${ }^{1}$ Department of Physics, Beijing Normal University, Beijing 100875, China \\ ${ }^{2}$ George P. \& Cynthia Woods Mitchell Institute for Fundamental Physics and Astronomy, \\ Texas AEMM University, College Station, TX 77843, USA \\ ${ }^{3}$ DAMTP, Centre for Mathematical Sciences, Cambridge University, \\ Wilberforce Road, Cambridge CBз OWA, UK
}

\begin{abstract}
$\underline{\text { ABSTRACT }}$
We obtain spherically-symmetric and $\mathbb{R}^{2}$-symmetric dyonic black holes that are asymptotic to anti-de Sitter space-time (AdS), which are solutions in maximal gauged fourdimensional supergravity, with just one of the $U(1)$ fields carrying both the electric and magnetic charges $(Q, P)$. We study the thermodynamics, and find that the usually-expected first law does not hold unless $P=0, Q=0$ or $P=Q$. For general values of the charges, we find that the first law requires a modification with a new pair of thermodynamic conjugate variables. We show that they describe the scalar hair that breaks some of the asymptotic AdS symmetries.
\end{abstract}




\section{Contents}

1 Introduction 1

$2 \quad S^{2}$-symmetric Dyonic AdS Black Hole 4

2.1 Local solutions and conserved quantities . . . . . . . . . . . . . . . 4

2.2 Thermodynamics . . . . . . . . . . . . . . . . . . . 6

2.3 Euclidean action and free energy . . . . . . . . . . . . . 8

2.4 Entropy product formula . . . . . . . . . . . . . . . . . . 10

$3 \quad \mathbb{R}^{2}$-Symmetric Dyonic AdS Black Hole 11

4 Limits of The Gauged Dyonic Black Hole 12

5 Lifting to M-theory 13

6 Thermodynamics and the Wald Canonical Charge 14

7 Conclusions 17

\section{Introduction}

The spherically-symmetric dyonic black hole of the four-dimensional Einstein-MaxwellDilaton theory obtained by the Kaluza-Klein reduction of five-dimensional pure gravity has some intriguing properties [1, 2, 3, 4, 5, 6. The solution can be obtained by means of a solution-generating technique, by performing a further $S^{1}$ reduction of the four-dimensional theory, which yields a scalar $S L(3, \mathbb{R}) / O(3)$ sigma model in three dimensions. Acting on the reduction of an uncharged black hole with an appropriate $O(1,1) \times O(1,1) \in S L(3, \mathbb{R})$ transformation then yields the dyonic black hole after lifting back to four dimensions. One can alternatively construct the solution by solving the equations of motion directly; they can be reduced to a set of $S L(3, \mathbb{R})$ Toda equations, which can be solved exactly [7]. The generalization to arbitrary dimensions and for an arbitrary $S L(n, \mathbb{R})$ Toda system were given recently in [8]. In the extremal limit the near-horizon geometry is $\mathrm{AdS}_{2} \times S^{2}$, and the mass is related to the charges by the somewhat unusual formula

$$
M=\left(P^{2 / 3}+Q^{2 / 3}\right)^{3 / 2} .
$$

Thus the black hole describes a bound state of electric and magnetic monopoles with negative binding energy. Although the solution is not supersymmetric, the entropy $S=8 \pi P Q$ 
in the extremal limit can be derived from the attractor mechanism [9], which is analogous to the procedure for supersymmetric solutions. In fact, the entropy of a general extremal charged black hole in the STU model is given by the triality-invariant expression of the form [10, 11] $S=8 \pi \sqrt{|D(p, q)|}$, where

$$
D(p, q)=4\left[\left(p^{1} q_{1}\right)\left(p^{2} q_{2}\right)+\left(p^{1} q_{1}\right)\left(p^{3} q_{3}\right)+\left(p^{2} q_{2}\right)\left(p^{3} q_{3}\right)-p^{0} q_{1} q_{2} q_{3}+q_{0} p^{1} p^{2} p^{3}\right]-\left(p^{\mu} q_{\mu}\right)^{2}
$$

where $q_{\mu}$ are the electric charges and $p^{\mu}$ the magnetic charges for the four gauge fields. The last term gives the contribution for the Kaluza-Klein dyon. The solution can be lifted to become a D0/D6 brane in string theory, or a pp-wave/NUT intersection in M-theory.

In this paper, we consider the Kaluza-Klein theory with an added scalar potential. To be more precise, the theory we consider can be embedded into maximal gauged supergravity in four dimensions. Maximal gauged supergravity in four dimensions has an $S O(8)$ gauge group, which has a $U(1)^{4}$ Cartan subgroup. In this paper, we set all except one of these $U(1)$ gauge fields to zero. The minimal bosonic field content then involves the metric, a dilaton $\phi$ and the vector field $A_{\mu}$. The relevant Lagrangian is given by

$$
\mathcal{L}=\sqrt{-g}\left[R-\frac{1}{2}(\partial \phi)^{2}-\frac{1}{4} e^{-\sqrt{3} \phi} F^{2}+6 g^{2} \cosh \left(\frac{1}{\sqrt{3}} \phi\right)\right]
$$

where $F=d A$. (There should be no confusion between the gauge coupling constant $g$ and the determinant of the metric.) The scalar potential has a stationary point at $\phi=0$, giving rise to a supersymmetric four-dimensional anti-de Sitter $\left(\mathrm{AdS}_{4}\right)$ vacuum, with $\mathrm{AdS}$ radius $\ell=1 / g$. If we set the gauge coupling constant $g$ to zero, the theory is the standard Kaluza-Klein theory obtained from the $S^{1}$ reduction of pure gravity in five dimensions. For non-vanishing $g$, the theory can be obtained as a consistent truncation of the $S^{7}$ reduction of eleven-dimensional supergravity.

Although the theory (1.3) cannot be supersymmetrised on its own, it can be "pseudosupersymmetrised." In other words, by adding a pseudo-gravitino and pseudo-dilatino, the Lagrangian can be made invariant, up to quadratic order in fermions, under pseudosupersymmetry transformations. This conclusion can be generalized to the Kaluza-Klein theory in an arbitrary dimension, for a specific choice of the scalar potential [12, 13]. In $D=4,5,6$ and 7 dimensions, the pseudo-supersymmetry transformation rules coincide with those in known supergravities in those dimensions, since the theories can then all be embedded in the relevant supergravities.

In section 2, we present the local spherically-symmetric solution. It is asymptotically AdS, and it contains three non-trivial parameters, characterising the mass $M$, and the 
electric and magnetic charges $Q$ and $P$. It should be emphasised that there is no continuous duality rotation symmetry in the theory, and so the generic dyonic black hole cannot be simply rotated into a purely electric or magnetic one.

By looking at the asymptotic form of the metrics, we obtain expressions for the conserved quantities $M, Q$ and $P$ in terms of the original parameters in the local metric. We then investigate the thermodynamics, and we find that the naive form $d M=T d S+\Phi_{Q} d Q+$ $\Phi_{P} d P$ of the first law is not obeyed unless one or another of the following conditions holds:

$$
\begin{array}{cll}
\text { Case I : } & g=0, \quad \text { for general }(Q, P) ; \\
\text { Case II : } & g \neq 0, \quad \text { and } \quad Q=0 \text { or } P=0 \text { or } Q=P .
\end{array}
$$

For the general AdS dyons, we find that it is necessary to introduce a new thermodynamic quantity $Y$ and its conjugate $X$, so that

$$
d M=T d S+\Phi_{Q} d Q+\Phi_{P} d P+X d Y
$$

in order to obtain a first law. We also verify that the product of the "entropies" at all of the horizons obeys a relation that is in accordance with general results seen in other black hole example, depending only on the quantised charges $Q$ and $P$ and the gauge coupling $g$.

In section 3, we take an appropriate limit to obtain the AdS dyonic black hole with a flat $\mathbb{R}^{2}$ horizon rather than the original $S^{2}$. The asymptotic form of the metric is then AdS in Poincaré planar coordinates. Again we find that the first law of thermodynamics requires the introduction of the additional conjugate pair $(X, Y)$ of thermodynamic variables.

In section 4 , we consider various limits of the AdS dyons. The extremal limit gives rise to near-horizon geometries that are either $\mathrm{AdS}_{2} \times S^{2}$ or $\mathrm{AdS}_{2} \times \mathbb{R}^{2}$. For the sphericallysymmetric solution, the "BPS"-like limit, which would give an extremal black hole in the case that $g=0$, now gives rise to a solution with a naked singularity. For the $\mathbb{R}^{2}$-symmetric solution, the gauge potential vanishes in the extremal limit, giving rise to a domain wall (membrane) solution supported by the potential for the scalar field. In section 5 , we study the lift of the solutions to $D=11$, via the consistent $S^{7}$ reduction.

In section 6 , we investigate the origin of the thermodynamic pair $(X, Y)$ that we had to introduce in the first law. By studying the asymptotics, we find that the fall-off of the scalar fields breaks some of the asymptotic AdS symmetries, except in the special cases enumerated in (1.4) for which the extra variables $X$ and $Y$ are not needed. We then employ Wald's procedure and obtain a scalar potential and charge, which are precisely the quantities $(X, Y)$ that are needed for establishing the first law of thermodynamics. We conclude the paper with comments in section 7 . 


\section{$2 \quad S^{2}$-symmetric Dyonic AdS Black Hole}

In this section we obtain the spherically-symmetric dyonic black hole solution of the theory described by (1.3), carrying both electric and magnetic charges. We shall first present the local solution and then discuss its thermodynamics.

\subsection{Local solutions and conserved quantities}

We find that the Lagrangian (1.3) admits the following static solution

$$
\begin{aligned}
d s^{2} & =-\left(H_{1} H_{2}\right)^{-\frac{1}{2}} f d t^{2}+\left(H_{1} H_{2}\right)^{\frac{1}{2}}\left(\frac{d r^{2}}{f}+r^{2}\left(d \theta^{2}+\sin ^{2} \theta d \varphi^{2}\right)\right), \\
\phi & =\frac{\sqrt{3}}{2} \log \frac{H_{2}}{H_{1}}, \quad f=f_{0}+g^{2} r^{2} H_{1} H_{2}, \quad f_{0}=1-\frac{2 \mu}{r}, \\
A & =\sqrt{2}\left(\frac{\left(1-\beta_{1} f_{0}\right)}{\sqrt{\beta_{1} \gamma_{2}} H_{1}} d t+2 \mu \gamma_{2}^{-1} \sqrt{\beta_{2} \gamma_{1}} \cos \theta d \varphi\right), \\
H_{1} & =\gamma_{1}^{-1}\left(1-2 \beta_{1} f_{0}+\beta_{1} \beta_{2} f_{0}^{2}\right), \quad H_{2}=\gamma_{2}^{-1}\left(1-2 \beta_{2} f_{0}+\beta_{1} \beta_{2} f_{0}^{2}\right), \\
\gamma_{1} & =1-2 \beta_{1}+\beta_{1} \beta_{2}, \quad \gamma_{2}=1-2 \beta_{2}+\beta_{1} \beta_{2} .
\end{aligned}
$$

The solution is written in a form where the parameters $\beta_{i}$ that characterise the charges enter in a symmetrical way, as was done in [8] for the $g=0$ (ungauged) case. The charge is purely magnetic if we set $\beta_{1}=0$, and purely electric if instead we set $\beta_{2}=0$. If we set $\beta_{1}=\beta_{2}$, the dilaton decouples and the metric becomes a dyonic Reissner-Nordström AdS black hole.

For the general case, one must require that $\beta_{i}>0$ and $\gamma_{i} \geq 0$ for it to be well behaved from the horizon out to infinity. This implies that one cannot freely choose the ranges of $\beta_{1}$ and $\beta_{2}$ independently. A different parameterisation, in terms of two independentlyspecifiable quantities $\lambda_{1}$ and $\lambda_{2}$, is given by writing

$$
\beta_{i}=\lambda_{j}^{-1}\left(1-\sqrt{1-\lambda_{1} \lambda_{2}}\right), \quad i \neq j,
$$

The parameters $\lambda_{i}$ can then independently take values lying in the range $0 \leq \lambda_{i} \leq 1$.

Our construction of this solution proceeded as follows. We first considered the case with vanishing gauge coupling constant, $g=0$. This Kaluza-Klein dyon has long been known. It turns out that the equations of motion for static black holes can be reduced to a system of one-dimensional $S L(3, \mathbb{R})$ Toda equations, which are completely solvable. (See, for example, [7, 8.) The general solution contains four integration constants. One of them is associated with a non-trivial scalar deformation and this has to be removed since it either gives rise to a naked curvature singularity or else it destroys the asymptotic flatness of the space-time. We adopted the notation of [8] in presenting the solution, and 
the parameters $\left(\mu, \beta_{1}, \beta_{2}\right)$ can be thought of as characterising the mass and the electric and magnetic charges. An alternative approach for constructing the ungauged dyonic black hole is to use a solution-generating technique. For $g=0$, the $S^{1}$ reduction of the Lagrangian (1.3), after dualising the Kaluza-Klein vector to an axion, gives a non-linear sigma model for the coset $S L(3, \mathbb{R}) / S O(3)$. Starting from an uncharged four-dimensional black hole, and reducing it to three dimensions, one then uses the two $O(1,1)$ boosts in $S L(3, \mathbb{R})$ to generate a new solution which, after lifting back to four dimensions, describes the dyonic black hole with electric and magnetic charges. This technique can be used to construct rotating dyonic black holes, as first obtained in [6], but for our purposes we need only the static case. The two $O(1,1)$ boost parameters $\delta_{i}$ are related to the $\beta_{i}$ we are using here by 1

$$
\sinh ^{2} \delta_{1}=\frac{2 \beta_{1} \gamma_{2}}{\left(1+\beta_{1} \beta_{2}\right) \gamma_{1}}, \quad \sinh ^{2} \delta_{2}=\frac{2 \beta_{2}}{\gamma_{2}},
$$

There is no solution-generating technique available for constructing the solution in the gauged theory. However, we found that having obtained the solution with $g=0$, we can simply replace the function $f_{0}$ by $f$ in the metric in order to obtain the solution in the $g \neq 0$ theory. This construction is analogous to that in other charged static AdS black holes in gauged supergravities [14, 15, 16, 17] and in certain general classes of non-supersymmetric theory [18].

The solution is asymptotic to AdS written in global coordinates. The mass can be easily calculated using the AMD method developed in [19, 20], by studying the asymptotic fall-off of the Weyl tensor on the conformal boundary (see also [21] for a discussion of the AMD procedure for asymptotically AdS black holes in supergravities). We find that it is given by

$$
M=\frac{\left(1-\beta_{1}\right)\left(1-\beta_{2}\right)\left(1-\beta_{1} \beta_{2}\right) \mu}{\gamma_{1} \gamma_{2}} .
$$

The electric and magnetic charges $Q$ and $P$ can be obtained straightforwardly by integrating the conserved quantities, giving

$$
Q=\frac{\mu \sqrt{\beta_{1} \gamma_{2}}}{\sqrt{2} \gamma_{1}}, \quad P=\frac{\mu \sqrt{\beta_{2} \gamma_{1}}}{\sqrt{2} \gamma_{2}} .
$$

Note that these conserved charges are independent of the gauge coupling constant $g$ and are the same as those given in [8] for the case $g=0$.

For later purpose, it is instructive to study the asymptotic fall-off. To do this, we introduce a new radial coordinate $\rho$, such that the metric becomes

$$
d s^{2}=-h d t^{2}+\tilde{h}^{-1} d \rho^{2}+\rho^{2} d \Omega_{2}^{2} .
$$

\footnotetext{
${ }^{1}$ In the solution-generating process it is necessary also to make a compensating $U(1)$ transformation to remove an unwanted NUT charge, and this results eventually in a somewhat awkward parameterisation in terms of the boost parameters $\delta_{i}$, which is why we prefer to use the $\beta_{i}$ parameterisation here.
} 
For large $\rho$, we find

$$
\begin{aligned}
h= & g^{2} \rho^{2}+1-\frac{2 M}{\rho}+\frac{4\left(Q^{2}+P^{2}\right)}{\rho^{2}}+\cdots, \\
\tilde{h}= & g^{2} \rho^{2}+1+\frac{3 g^{2} \mu^{2}\left(\beta_{1}-\beta_{2}\right)^{2}\left(1-\beta_{1} \beta_{2}\right)^{2}}{\gamma_{1}^{2} \gamma_{2}^{2}} \\
& -\frac{1-\beta_{1} \beta_{2}}{\gamma_{1} \gamma_{2}}\left[2 \mu\left(1-\beta_{1}\right)\left(1-\beta_{2}\right)+\frac{8 g^{2} \mu^{3}\left(\beta_{1}-\beta_{2}\right)^{2} \gamma}{\gamma_{1}^{2} \gamma_{2}^{2}}\right] \frac{1}{\rho}+\cdots, \\
= & h\left[1+\frac{3 \mu^{2}\left(\beta_{1}-\beta_{2}\right)^{2}\left(1-\beta_{1} \beta_{2}\right)^{2}}{\gamma_{1}^{2} \gamma_{2}^{2} \rho^{2}}-\frac{8 \mu^{3}\left(\beta_{1}-\beta_{2}\right)^{2}\left(1-\beta_{1} \beta_{2}\right) \gamma}{\gamma_{1}^{3} \gamma_{2}^{3} \rho^{3}}+\cdots\right],
\end{aligned}
$$

where the constant $\gamma$ is defined by

$$
\gamma \equiv \beta_{1}+\beta_{2}-8 \beta_{1} \beta_{2}+6 \beta_{1}^{2} \beta_{2}+6 \beta_{1} \beta_{2}^{2}-8 \beta_{1}^{2} \beta_{2}^{2}+\beta_{1}^{3} \beta_{2}^{2}+\beta_{1}^{2} \beta_{2}^{3} .
$$

The scalar at large $\rho$ is given by

$$
\cosh \left(\frac{\phi}{\sqrt{3}}\right)=1+\frac{2 \mu^{2}\left(\beta_{1}-\beta_{2}\right)^{2}\left(1-\beta_{1} \beta_{2}\right)^{2}}{\gamma_{1}^{2} \gamma_{2}^{2} \rho^{2}}-\frac{4 \mu^{3}\left(\beta_{1}-\beta_{2}\right)^{2}\left(1-\beta_{1} \beta_{2}\right) \gamma}{\gamma_{1}^{3} \gamma_{2}^{3} \rho^{3}}+\cdots
$$

The scalar $\phi$ vanishes if $\beta_{1}=\beta_{2}$, and consequently we have $h=\tilde{h}$ and the metric becomes that of the Reissner-Nordström black hole. (The scalar does not vanish for $\beta_{1} \beta_{2}=1$, which corresponds to a naked singularity.)

\subsection{Thermodynamics}

In the previous subsection, we calculated the mass and the electric and magnetic charges of the gauged dyonic solution. These quantities were determined from the asymptotic form of the solution near infinity, irrespective of whether the solution had a regular black hole horizon or not. For the solution to describe a black hole, the parameters must be restricted so that the metric describes an event horizon at some radius $r=r_{0}$. All the fields, including the metric, gauge field and scalar, should be well behaved from $r=r_{0}$ to $r \rightarrow \infty$. The horizon is defined to be the largest (real) root of the function $f$, namely

$$
1-\frac{2 \mu}{r_{0}}+g^{2} r_{0}^{2} H_{1}\left(r_{0}\right) H_{2}\left(r_{0}\right)=0 .
$$

Note that for $g=0$, the outer horizon is simply located at $r_{0}=2 \mu$, in which case the inner horizon is at $r=0$. For non-vanishing $g$, none of the the quantities $\beta_{i}, \mu$ or $r_{0}$ can conveniently be solved for in closed form. Furthermore, the surface at $r=0$ plays no distinguished role, and it does not correspond to a null surface.

The temperature and the entropy associated with the outer horizon can be obtained using standard techniques, and are given by

$$
T=\frac{f^{\prime}\left(r_{0}\right)}{4 \pi \sqrt{H_{1}\left(r_{0}\right) H_{2}\left(r_{0}\right)}}, \quad S=\pi r_{0}^{2} \sqrt{H_{1}\left(r_{0}\right) H_{2}\left(r_{0}\right)} .
$$


The calculation of the electric potential $\Phi_{Q}$ is straightforward; it is given by the time component of the potential $A_{\mu} d x^{\mu}$. The magnetic potential $\Phi_{P}$ can be obtained by performing a Hodge dualisation, which exchanges the roles of the electric and magnetic charges. The magnetic potential is then given by the time component of the vector potential for the dual field. Thus we find that the electric and magnetic potentials, which vanish at infinity, are given on the horizon by

$$
\begin{aligned}
\Phi_{Q} & =\sqrt{\frac{2}{\beta_{1} \gamma_{2}}}\left(1-\beta_{1}-\frac{1-\beta_{1} f_{0}\left(r_{0}\right)}{H_{1}\left(r_{0}\right)}\right), \\
\Phi_{P} & =\sqrt{\frac{2}{\beta_{2} \gamma_{1}}}\left(1-\beta_{2}-\frac{1-\beta_{2} f_{0}\left(r_{0}\right)}{H_{2}\left(r_{0}\right)}\right) .
\end{aligned}
$$

One might expect that the first law of thermodynamics should be just $d M=T d S+$ $\Phi_{Q} d Q+\Phi_{P} d P$, but, as mentioned in the introduction, this is not in general the case, with the exceptions listed in (1.4). In particular, it does hold for generic electric and magnetic charges provided that the gauge-coupling $g$ is zero. This is to be expected, since the thermodynamics of the asymptotically flat Kaluza-Klein dyon has long been understood. (See, for example, [6].) For non-vanishing $g$, we find the first law holds only if $\beta_{1}=0$, corresponding to $Q=0$; or if $\beta_{2}=0$, corresponding to $P=0$; or if $\beta_{1}=\beta_{2}$, corresponding to $Q=P$. In order for the first law to work for generic choices of the charges, we must introduce an additional intensive/extensive conjugate pair of thermodynamic variables $(X, Y)$, and modify the first law to become

$$
d M=T d S+\Phi_{Q} d Q+\Phi_{P} d P+X d Y
$$

By solving for $X$ and $Y$ such that this modified law holds, we find that $X$ and $Y$ can be taken to be

$$
X=\frac{4 g^{2} \mu^{3}\left(\beta_{1}-\beta_{2}\right) \sqrt{\beta_{1} \beta_{2}^{3}}}{\left(1-\beta_{1} \beta_{2}\right) \gamma_{2}^{2}}, \quad Y=\frac{\sqrt{\beta_{1}} \gamma_{2}}{\sqrt{\beta_{2}} \gamma_{1}},
$$

There is actually a certain freedom in our choice of the functions $X$ and $Y$. We can, accordingly, replace $Y$ by any function of $Y$, provided we then make the corresponding appropriate adjustment to $X$. Although $Y$ becomes singular when $\beta_{2}=0$, the quantity $X d Y$ vanishes and hence the extra "hair" does not contribute to the thermodynamics in this case. In fact, it is easy to see that $X d Y$ has a zero contribution if either $g=0$, or else, when $g \neq 0$, if $\beta_{1}=0$ or $\beta_{2}=0$ or $\beta_{1}=\beta_{2}$. In other words, $X d Y$ plays no role in any of the cases enumerated in (1.4) for which the usual first law holds. We shall give a detailed discussion of the significance of the $X$ and $Y$ variables in section 6 .

We can also go one step further, and treat the cosmological constant $\Lambda=-3 g^{2}$ of the asymptotically-AdS metric as an additional thermodynamic quantity like a pressure, with 
its conjugate "potential" $\Upsilon$, such that

$$
d M=T d S+\Phi_{Q} d Q+\Phi_{P} d P+X d Y+\Upsilon d \Lambda
$$

We find that $\Upsilon$, which can be interpreted as a volume (see, for example, 22] and references therein), is given by

$$
\begin{gathered}
\Upsilon=\frac{r_{0}^{3}}{12 \gamma_{1} \gamma_{2}}\left(\beta_{1} \beta_{2}\left(-\beta_{1}-\beta_{2}+2 \beta_{1} \beta_{2}\right) \tilde{f}_{0}^{3}-3 \beta_{1} \beta_{2}\left(2-\beta_{1}-\beta_{2}\right) \tilde{f}_{0}^{2}\right. \\
\left.+3\left(\beta_{1} \beta_{2}-2 \beta_{1} \beta_{2}\right) \tilde{f}_{0}-2+\beta_{1}+\beta_{2}\right)
\end{gathered}
$$

where $\tilde{f}_{0}=1-\mu / r_{0}$.

If we define a thermodynamic potential

$$
\Phi_{\text {thermo }} \equiv M-T S-\Phi_{Q} Q-\Phi_{P} P
$$

then we find it is given simply by

$$
\Phi_{\text {thermo }}=\frac{1}{2}\left(r_{0}-\mu\right) .
$$

It does not explicitly depend on $g$ and $\beta_{i}$, a feature that appears to be a rather universal for the general class of $S L(n, \mathbb{R})$ black holes considered in [8]. We also find that the Smarr formula is given by

$$
M=2 T S+\Phi_{Q} Q+\Phi_{P} P-2 \Upsilon \Lambda .
$$

Note that, given the first law (2.15), the coefficient of the terms in (2.19) follow just from the scaling dimensions of the thermodynamic quantities, and in particular, since $Y$ is dimensionless, $X Y$ does not appear in the Smarr formula.

\subsection{Euclidean action and free energy}

We may compute the Euclidean action by using the holographic renormalization method described in [23]. The divergences in the classical on-shell Euclidean action are removed by the addition of local covariant surface counterterms. In our case, the renormalized action in Lorentzian signature is given by

$$
I=I_{\text {bulk }}+I_{\text {surf }}+I_{\text {ct }}
$$

where

$$
\begin{aligned}
I_{\text {bulk }} & =\frac{1}{16 \pi G} \int_{\mathcal{M}} d^{4} x \sqrt{-g}\left(R-\frac{1}{2}(\partial \phi)^{2}-\frac{1}{4} e^{-\sqrt{3} \phi} F^{2}+6 g^{2} \cosh \left(\frac{1}{\sqrt{3}} \phi\right)\right) \\
I_{\text {surf }} & =-\frac{1}{8 \pi G} \int_{\partial \mathcal{M}} d^{3} x \sqrt{-h} K
\end{aligned}
$$


and the counterterms take the form

$$
I_{\mathrm{ct}}=-\frac{1}{8 \pi G} \int_{\partial \mathcal{M}} d^{3} x \sqrt{-h}\left(\frac{2}{\ell}+\frac{\ell}{2} \mathcal{R}\right)+\frac{1}{48 \pi G} \int_{\partial \mathcal{M}} d^{3} x \sqrt{-h}\left(\phi n^{\mu} \partial_{\mu} \phi-\frac{1}{2 \ell} \phi^{2}\right) .
$$

In the above equation, $K_{\mu \nu} \equiv-\frac{1}{2}\left(\nabla_{\mu} n_{\nu}+\nabla_{\nu} n_{\mu}\right)$ is the extrinsic curvature of the boundary surface, with $n_{\mu}$ being the outward unit normal vector. $\ell=1 / g$ is the curvature radius of AdS, and $\mathcal{R}$ is the Ricci scalar of the boundary metric.

Using these counterterms, the renormalized energy-momentum tensor is given by $T^{\mu \nu} \equiv$ $(2 / \sqrt{-h}) \delta I / \delta h_{\mu \nu}$, yielding

$$
T_{\mu \nu}=\frac{1}{8 \pi G}\left(K_{\mu \nu}-K h_{\mu \nu}-\frac{2}{\ell} h_{\mu \nu}+\ell\left(\mathcal{R}_{\mu \nu}-\frac{1}{2} \mathcal{R} h_{\mu \nu}\right)+\frac{1}{6} h_{\mu \nu}\left(\phi n^{\rho} \partial_{\rho} \phi-\frac{1}{2 \ell} \phi^{2}\right)\right) .
$$

In cases where the boundary geometry possesses a timelike Killing vector, one can define the energy of the dual field theory, which is equal to the energy of the black hole via the AdS/CFT correspondence.

We parameterise the boundary metric as a foliation of spacelike surfaces $\Sigma$ with metric $\sigma_{a b}:$

$$
h_{\mu \nu} d x^{\mu} d^{\nu}=-N^{2} d t^{2}+\sigma_{a b}\left(d x^{a}+N^{a} d t\right)\left(d x^{b}+N^{b} d t\right) .
$$

The energy can be calculated by evaluating

$$
M=\int_{\Sigma} d^{2} x \sqrt{\sigma} N u^{\mu} u^{\nu} T_{\mu \nu}
$$

where $u^{\mu}$ is the the unit timelike normal to $\Sigma$. Evaluating this for the AdS dyon (2.1), we recover precisely the AMD expression for the mass that we obtained in (2.4).

By definition the Euclidean action $I_{E}$ is given by the Euclideanisation of $-I$ defined in (2.20), and by standard arguments [24] this is equal to $\beta\left(\left.\mathcal{H}\right|_{\infty}-\left.\mathcal{H}\right|_{r_{0}}\right)$, where $\left.\mathcal{H}\right|_{\infty}$ and $\left.\mathcal{H}\right|_{r_{0}}$ are the values of the Hamiltonian at infinity and at the horizon $r=r_{0}$. Since our electrostatic potential is chosen to vanish at infinity we then find

$$
\left.\mathcal{H}\right|_{\infty}=M,\left.\quad \mathcal{H}\right|_{r_{0}}=T S+\Phi_{Q} Q
$$

and hence we arrive at the result that

$$
T I_{E}=M-T S-\Phi_{Q} Q
$$

We see, in particular, that the thermodynamic quantities $X$ and $Y$ do not enter in this relation.

It should be noted also that while the electric charge and electric potential enter in the free energy on the right-hand side of (2.27), the magnetic charge and magnetic potential do 
not. We obtained this result using the conventional definition of the renormalised action (2.20). One could always add an additional surface term,

$$
I_{\text {extra }}=\frac{1}{16 \pi G} \int_{\partial M} \sqrt{-h} d^{3} x e^{-\sqrt{3} \phi} n_{\mu} F^{\mu \nu} A_{\nu}
$$

which would correspond to changing the boundary conditions in the variational problem from the case where $A_{\mu}$ is specified on $\partial M$ to the case where instead $F_{\mu \nu}$ is specified. This would have the effect of removing the $\Phi_{Q} Q$ term in (2.27). (See [25] for a related discussion.) This amounts to performing a Legendre transformation on the free energy that appears on the right-hand side of (2.27), to a new energy for which $Q$, rather than $\Phi_{Q}$, is held fixed in the first law. Note that the thermodynamic potential defined in eqn (2.17) corresponds to a different Legendre transformation of the free energy in (2.27). This one, however, could not be achieved by adding a surface term to the renormalised action (2.20) that was a local function of the potential $A_{\mu}$.

\subsection{Entropy product formula}

When $g=0$, there are two null surfaces, located at $r_{+}=2 \mu$ and $r_{-}=0$. If we introduce the concept of an entropy for each null surface, or horizon, as one quarter of the area of the horizon, one can see that [26]

$$
S_{+} S_{-}=\frac{1}{4} \pi^{2} P^{2} Q^{2}
$$

which is quantised and independent of the mass parameter.

Analogous entropy product formulae exist for all the $S L(n, \mathbb{R})$ Toda black holes [8]. The first observation of such a property was given in [27]. When $g \neq 0$ the metric function $f$ has in general four roots, each corresponding to a null surface. It was argued that a universal entropy product formula should still hold if one considers the product of the entropies of all null surfaces [28]. Indeed, we find that the result in the present case is independent of the mass:

$$
\prod_{i=1}^{4} S_{i}=\frac{64 \pi^{4}}{g^{4}} P^{2} Q^{2} .
$$

This result can be obtained by noting from (2.1) that

$$
S_{i}^{2}=\left.\pi^{2}\left(r^{4} H_{1} H_{2}\right)\right|_{r=r_{i}}=\frac{\pi^{2}}{g^{2}}\left(2 \mu-r_{i}\right) r_{i},
$$

where $r_{i}$ is the radius of the $i$ 'th null surface, which is one of the four roots of $f\left(r_{i}\right)=0$. Thus it follows that $\prod_{i} S_{i}^{2}$ is expressible in terms of $\mu$ and the four symmetric multinomials

$$
\Delta_{4}=\prod_{i} r_{i}, \quad \Delta_{3}=r_{1} r_{2} r_{3}+\cdots, \quad \Delta_{2}=r_{1} r_{2}+\cdots, \quad \Delta_{1}=\sum_{i} r_{i}
$$


Since $f\left(r_{i}\right)=0$ for each $i$ it follows that the $\Delta_{i}$ are expressible purely in terms of the parameters $\beta_{1}, \beta_{2}$ and $\mu$ on which $f$ depends, and so $\prod_{i} S_{i}$ is necessarily expressible as a function only of $\beta_{1}, \beta_{2}$ and $\mu$. Comparing with the expressions given in (2.5), it then turns out that when expressed in terms of $Q$ and $P$, the product of entropies is independent of $\mu$.

\section{$3 \quad \mathbb{R}^{2}$-Symmetric Dyonic AdS Black Hole}

In the previous section we constructed the static spherically-symmetric black hole gauged dyon. Since the solutions are asymptotic to AdS, there will also exist solutions whose level surfaces, and horizon, are flat $\mathbb{R}^{2}$, or hyperbolic. These so called "topological" black holes can easily be obtained from the previous $S^{2}$ black holes by making an appropriate scaling of the parameters, namely

$$
r \rightarrow \epsilon^{-1 / 2} r, \quad t \rightarrow \epsilon^{1 / 2} t \quad \beta_{i} \rightarrow \epsilon \beta_{i}, \quad \mu \rightarrow \epsilon^{-3 / 2} \mu, \quad d \Omega_{2}^{2} \rightarrow \epsilon d \Omega_{2, \epsilon}^{2} .
$$

Taking $\epsilon=-1$ gives the black hole with hyperbolic horizon. The limit where $\epsilon=0$ gives the $\mathbb{R}^{2}$ black hole, which is of particular interest because the asymptotically AdS region is then written in Poincaré coordinates, and hence is particularly suited for the discussion of the AdS/CFT correspondence. The solution is given by

$$
\begin{aligned}
d s^{2} & =-\left(H_{1} H_{2}\right)^{-\frac{1}{2}} f d t^{2}+\left(H_{1} H_{2}\right)^{\frac{1}{2}}\left(\frac{d r^{2}}{f}+r^{2}\left(d x^{2}+d y^{2}\right)\right), \\
\phi & =\frac{\sqrt{3}}{2} \log \frac{H_{2}}{H_{1}}, \quad f=-\frac{2 \mu}{r}+g^{2} r^{2} H_{1} H_{2}, \\
A & =\sqrt{2 \mu}\left(\frac{\left(r+2 \beta_{1}\right)}{\sqrt{\beta_{1}} H_{1} r} d t+2 \sqrt{\beta_{2}} x d y\right), \\
H_{1} & =1+\frac{4 \beta_{1}}{r}+\frac{4 \beta_{1} \beta_{2}}{r^{2}}, \quad H_{2}=1+\frac{4 \beta_{2}}{r}+\frac{4 \beta_{1} \beta_{2}}{r^{2}},
\end{aligned}
$$

In presenting this $\epsilon=0$ solution, we have made a further redefinition $\beta_{i} \rightarrow \beta_{i} / \mu$. This new parameterisation is convenient because then $H_{1}$ and $H_{2}$ are independent of $\mu$, and so one can then solve linearly for $\mu$ in terms of the horizon radius $r=r_{0}$, namely

$$
\mu=\frac{1}{2} g^{2} r_{0}^{3} H_{1}\left(r_{0}\right) H_{2}\left(r_{0}\right),
$$

The temperature and the entropy then take the same form as in (2.11). Here we are, for convenience, assuming that the $\mathbb{R}^{2}$ coordinates $(x, y)$ have been identified to give a 2-torus of volume $4 \pi$. One can take any other choice for the volume, with the understanding that the extensive quantities should be scaled by the relative volume factor. We find that the remaining thermodynamic quantities are

$$
M=\mu, \quad Q=\sqrt{\frac{\mu \beta_{1}}{2}}, \quad P=\sqrt{\frac{\mu \beta_{2}}{2}},
$$




$$
\begin{aligned}
& \Phi_{Q}=\frac{2 \sqrt{2 \mu \beta_{1}}\left(r_{0}+2 \beta_{2}\right)}{r_{0}^{2} H_{1}\left(r_{0}\right)}, \quad \Phi_{P}=\frac{2 \sqrt{2 \mu \beta_{2}}\left(r_{0}+2 \beta_{1}\right)}{r_{0}^{2} H_{2}\left(r_{0}\right)}, \\
& X=4 g^{2}\left(\beta_{1}-\beta_{2}\right) \sqrt{\beta_{1} \beta_{2}^{3}}, \quad Y=\sqrt{\frac{\beta_{1}}{\beta_{2}}}, \quad \Lambda=-3 g^{2}, \\
& \Upsilon=-\frac{1}{6}\left(4 \beta_{1} \beta_{2}\left(\beta_{1}+\beta_{2}\right)+12 \beta_{1} \beta_{2} r_{0}+3\left(\beta_{1}+\beta_{2}\right) r_{0}^{2}+r_{0}^{3}\right),
\end{aligned}
$$

It is now straightforward to verify that the first law of thermodynamics (2.15) is again satisfied. The Smarr formula takes the same form as (2.19) and the thermodynamic potential is now simply

$$
\Phi_{\text {thermo }}=-\frac{1}{2} \mu=-\frac{1}{2} M .
$$

The entropy product formula is the same as (2.30).

\section{Limits of The Gauged Dyonic Black Hole}

Having obtained the general solution, we may consider various limits of the parameters. The most obvious one to consider is the extremal limit, for which the function $f$ acquires a double zero at its largest root. The near-horizon geometry in this case then becomes $\mathrm{AdS}_{2} \times S^{2}$, or $\mathrm{AdS}_{2} \times \mathbb{R}^{2}$. The procedure for taking this limit is straightforward and we shall not present any explicit results.

Let us instead consider the "BPS"-type of "extremal" limit, in which we send $\mu$ to zero while keeping $Q$ and $P$ finite. (We refer to it as "BPS" because this limiting solution becomes supersymmetric in either of the cases $Q=0$ or $P=0$.) The limit can be taken by using the the parameterisation in terms of $\lambda_{i}$ introduced in (2.2), and defining

$$
\lambda_{i}=1-\frac{k^{2}}{b_{i}^{2}}, \quad \mu=2 k b_{1} b_{2},
$$

Taking the limit $k \rightarrow 0$ in the spherically-symmetric dyon (2.1) we then find $Q=b_{1}^{3}, P=b_{2}^{3}$ and $M=\left(b_{1}^{2}+b_{2}^{2}\right)^{3 / 2}$, and

$$
\begin{aligned}
H_{1} & =1+\frac{4 Q^{2 / 3} \sqrt{P^{2 / 3}+Q^{2 / 3}}}{r}+\frac{8 P^{2 / 3} Q^{4 / 3}}{r^{2}}, \\
H_{2} & =1+\frac{4 P^{2 / 3} \sqrt{P^{2 / 3}+Q^{2 / 3}}}{r}+\frac{8 P^{4 / 3} Q^{2 / 3}}{r^{2}}, \\
f & =r^{2}+g^{2} r^{4} H_{1} H_{2} .
\end{aligned}
$$

The vector potential is now given by

$$
A=\frac{Q\left(4 r+8 P^{2 / 3} \sqrt{P^{2 / 3}+Q^{2 / 3}}\right)}{r^{2}+4 Q^{2 / 3} \sqrt{P^{2 / 3}+Q^{2 / 3}} r+8 P^{2 / 3} Q^{4 / 3}} d t+4 P \cos \theta d \phi .
$$


If $g=0$, the horizon with the double root is located at $r=0$, and the near-horizon geometry is $\mathrm{AdS}_{2} \times S^{2}$. When $g \neq 0$, the radius $r=0$ has no particular significance, and the singularity, corresponding to having $H_{1} H_{2}=0$, becomes naked. The mass and the conjugate pair $(X, Y)$ are given by

$$
M=\left(P^{2 / 3}+Q^{2 / 3}\right)^{3 / 2}, \quad X=\frac{g^{2} P^{2} Q^{2 / 3}\left(Q^{2 / 3}-P^{2 / 3}\right)}{\sqrt{P^{2 / 3}+Q^{2 / 3}}}, \quad Y=\frac{Q^{2 / 3}}{P^{2 / 3}} .
$$

The situation is very different for the $\mathbb{R}^{2}$-symmetric AdS dyon (3.2). In this case, setting $\mu=0$ implies that the gauge potential vanishes, and the resulting solution becomes the AdS membrane

$$
d s^{2}=r^{2}\left(H_{1} H_{2}\right)^{\frac{1}{2}}\left(-g^{2} d t^{2}+d x^{2}+d y^{2}\right)+\left(H_{1} H_{2}\right)^{-\frac{1}{2}} \frac{d r^{2}}{g^{2} r^{2}}, \quad e^{\frac{2}{\sqrt{3}} \phi}=\frac{H_{2}}{H_{1}},
$$

where the $H_{i}$ are again given by (3.2).

\section{Lifting to M-theory}

The four-dimensional theory described by (1.3) can itself be embedded within the gauged four-dimensional supergravity STU model, whose bosonic embedding as a 7-sphere reduction of eleven-dimensional supergravity was given in [16] (see section 3 of that paper). The metric reduction ansatz is given by

$$
d \hat{s}_{11}^{2}=\tilde{\Delta}^{2 / 3} d s_{4}^{2}+g^{-2} \tilde{\Delta}^{-1 / 3} \sum_{i=1}^{4} X_{i}^{-1}\left(d \mu_{i}^{2}+\mu_{i}^{2}\left(d \phi_{i}+g A^{i}\right)^{2}\right),
$$

where $A^{i}$ are the four $U(1)$ gauge potentials, $X_{i}$ are exponentials of the dilatonic scalar fields (defined in [16])), and the four direction cosines $\mu_{i}$ satisfy $\sum_{i} \mu_{i}^{2}=1$. Writing these as

$$
\mu_{1}=\sin \tilde{\theta}, \quad \mu_{2}=\cos \tilde{\theta} \sin \tilde{\varphi}, \quad \mu_{3}=\cos \tilde{\theta} \cos \tilde{\varphi} \sin \tilde{\psi}, \quad \mu_{4}=\cos \tilde{\theta} \cos \tilde{\varphi} \cos \tilde{\psi},
$$

and substituting the scalar fields of our four-dimensional solution into the expressions for the $X_{i}$ given in [16], we find

$$
\begin{aligned}
X_{1} & =\left(\frac{H_{1}}{H_{2}}\right)^{-3 / 4}, \quad X_{2}=X_{3}=X_{4}=\left(\frac{H_{1}}{H_{2}}\right)^{1 / 4}, \\
\tilde{\Delta} & =H_{1}^{1 / 4} H_{2}^{3 / 4}\left(H_{1}^{-1} \sin ^{2} \tilde{\theta}+H_{2}^{-1} \cos ^{2} \tilde{\theta}\right),
\end{aligned}
$$

and the four gauge potentials are given by $A_{(1)}^{1}=A$ and $A_{(1)}^{2}=A_{(1)}^{3}=A_{(1)}^{4}=0$. The eleven-dimensional metric is therefore given by

$$
\begin{aligned}
d \hat{s}_{11}^{2}= & \tilde{\Delta}^{2 / 3} d s_{4}^{2}+4 g^{-2} \tilde{\Delta}^{2 / 3}\left(\frac{H_{1}}{H_{2}}\right)^{1 / 2} d \tilde{\theta}^{2} \\
& +4 g^{-2} \tilde{\Delta}^{-1 / 3}\left[\left(\frac{H_{1}}{H_{2}}\right)^{3 / 4} \sin ^{2} \tilde{\theta}\left(d \phi_{1}+\frac{1}{2} g A\right)^{2}+\left(\frac{H_{1}}{H_{2}}\right)^{-1 / 4} \cos ^{2} \tilde{\theta} d \Omega_{5}^{2}\right],
\end{aligned}
$$


where

$$
d \Omega_{5}^{2}=d \tilde{\varphi}^{2}+\sin ^{2} \tilde{\varphi} d \phi_{2}^{2}+\cos ^{2} \tilde{\varphi}\left(d \tilde{\psi}^{2}+\sin ^{2} \tilde{\psi} d \phi_{3}^{2}+\cos ^{2} \tilde{\psi} d \phi_{4}^{2}\right)
$$

is the metric on a unit 5 -sphere. Here, $d s_{4}^{2}$ and $A$ are the metric and gauge potential of the four-dimensional dyonic gauged black hole given in (2.1).

Using the expression in [16] for the 4-form field strength in eleven dimensions, we obtain

$$
\begin{aligned}
\hat{F}_{(4)}= & -g\left[2\left(\frac{H_{1}}{H_{2}}\right)^{1 / 2} \cos ^{2} \tilde{\theta}+\left(\frac{H_{1}}{H_{2}}\right)^{-1 / 2}\left(1+2 \sin ^{2} \tilde{\theta}\right)\right] \epsilon_{(4)} \\
& -2 g^{-1} \sin \tilde{\theta} \cos \tilde{\theta}\left(H_{1}^{-1} * d H_{1}-H_{2}^{-1} * d H_{2}\right) \wedge d \tilde{\theta} \\
& -4 g^{-2}\left(\frac{H_{1}}{H_{2}}\right)^{3 / 2} \sin \tilde{\theta} \cos \tilde{\theta} d \tilde{\theta} \wedge\left(d \phi_{1}+\frac{1}{2} g A\right) \wedge * F
\end{aligned}
$$

where $\epsilon_{(4)}$ is the volume-form of the four-dimensional metric and $*$ denotes the Hodge dual in the four-dimensional metric.

In the extremal limit, the metric $d s_{4}^{2}$ becomes $\operatorname{AdS}_{2} \times S^{2}$, which is the base space for

the fibre direction $d \phi_{1}+\frac{1}{2} g A$. For an appropriate Euclideanisation, the eleven-dimensional metric can be viewed as a foliation of $T_{p, q}$ and $S^{5}$.

\section{Thermodynamics and the Wald Canonical Charge}

Here we shall return to the intriguing feature that we found when studying the thermodynamics of the gauged dyonic black hole, namely that the naively-expected first law $d M=T d S+\Phi_{Q} d Q+\Phi_{P} d P$ turned out not to hold except in the special cases enumerated in (1.4). In general, we found that we had to introduce a new pair of thermodynamic conjugate variables $(X, Y)$ in the first law, as in (2.13). This is reminiscent of the situation for the thermodynamics of black holes in conformal gravity [29, 30]. However, in four-dimensional conformal gravity, the black hole solution 31] has an extra integration constant on account of the higher-derivative equations of motion, and hence it is natural in that case to have to introduce the notion of "massive spin-2 hair" with its associated charge and potential [29]. Our AdS dyon, on the other hand, has only three integration constants, which are related to the mass, and the electric and magnetic charges. Thus, the quantities $(X, Y)$ must be expressible as functions of $(M, P, Q)$. This implies that it would be possible to redefine the other thermodynamic quantities in such a way that a "standard" first law, with no $X$ and $Y$, would satisfied.

This can be seen most easily in the case of the $\mathbb{R}^{2}$-symmetric solution (3.2), whose thermodynamic quantities are given in (3.4). In this case, we see that $Y=Q / P$, and so if 
we redefine

$$
\Phi_{Q} \longrightarrow \widetilde{\Phi}_{q}=\Phi_{Q}-\frac{X}{P}, \quad \Phi_{P} \longrightarrow \widetilde{\Phi}_{P}=\Phi_{P}+\frac{X Q}{P^{2}}
$$

then the usual first law of thermodynamics would be recovered. However, these redefinitions are rather artificial, since the electric and magnetic potentials are ostensibly well-defined quantities with no apparent a priori motivation for such a modification. The situation is even less clear in the case of the $S^{2}$-symmetric solutions (2.1), since now $Y$ depends on $M$ as well as $Q$ and $P$, so the necessary redefinitions would require seemingly unmotivated modifications to other quantities in addition to $\Phi_{Q}$ and $\Phi_{P}$.

A more satisfactory explanation would be if $(X, Y)$ admitted a physical interpretation in their own right, as a scalar potential and charge. At first sight this might seem problematic since, as can be seen from the asymptotic behaviour of the metric and the scalar field, discussed in section 2, the characteristic behaviour for generic values of $(Q, P)$ appears to be the same as in either of the special cases $P=0$ and $Q=0$. And yet, the first law requires the introduction of $(X, Y)$ in the generic case but not in either of these special cases.

In order to settle this, we can use the procedure developed by Wald [32, 33] to determine whether there is some additional charge. Given the Lagrangian (1.3), one can calculate the Noether charge associated with a Killing vector $\xi$. Following [32, 33], the conserved charge takes the form

$$
\begin{aligned}
& \mathcal{Q}_{\xi}=\mathcal{Q}_{\xi}^{\text {gravity }}+\mathcal{Q}_{\xi}^{\mathrm{em}}, \\
& \mathcal{Q}_{\xi}^{\text {gravity }}=-\frac{1}{16 \pi G} * d \xi, \quad \mathcal{Q}_{\xi}^{\mathrm{em}}=-\frac{1}{16 \pi G}\left(i_{\xi} A\right) e^{-\sqrt{3} \phi} * F,
\end{aligned}
$$

where $i_{\xi}$ denotes the contraction of $\xi^{\mu}$ with a form field, and so $i_{\xi} A=\xi^{\mu} A_{\mu}$. In deriving this expression, we have chosen a gauge where $\mathcal{L}_{\xi} A_{\mu}=0$. On the other hand, if we consider the variations of $g_{\mu \nu}, A_{\mu}$ and $\phi$ on the solution space, we can define a closed 2-form whose differential, when integrated on the domain bounded by infinity and horizon, leads to the first law of thermodynamics. As explained in [32, the integration of the closed 2-form at infinity is identified as the infinitesimal Hamiltonian which generates the Hamiltonian flow associated with the vector field $\xi$. The infinitesimal Hamiltonian corresponding to the theory (1.3) is given as

$$
\delta \mathcal{H}=\int_{\infty}\left(\delta \mathcal{Q}_{\xi}-i_{\xi} \Theta\right), \quad i_{\xi} \Theta=i_{\xi} \Theta^{\text {gravity }}+i_{\xi} \Theta^{\mathrm{em}}+i_{\xi} \Theta^{\text {scalar }},
$$


where

$$
\begin{aligned}
i_{\xi} \Theta^{\text {gravity }} & =-\frac{1}{2} \epsilon_{\alpha \beta \mu \nu} \xi^{\alpha} \Xi^{\beta} d x^{\mu} \wedge d x^{\nu}, \quad \Xi^{\beta}=\frac{1}{16 \pi G}\left(g^{\mu \beta} g^{\alpha \nu}-g^{\mu \nu} g^{\alpha \beta}\right) \nabla_{\alpha} \delta g_{\mu \nu}, \\
i_{\xi} \Theta^{\mathrm{em}} & =-\frac{1}{16 \pi G}\left(\delta\left(i_{\xi} A\right) e^{-\sqrt{3} \phi} * F+\Psi \delta F\right), \quad d \Psi=e^{-\sqrt{3} \phi} i_{\xi} * F \\
i_{\xi} \Theta^{\text {scalar }} & =\frac{1}{16 \pi G} \delta \phi i_{\xi} * d \phi .
\end{aligned}
$$

Note that we are following the notation of Wald [32] here; the symbol $\delta \mathcal{H}$ is not intended to imply that it is necessarily an integrable variation of a Hamiltonian $\mathcal{H}$.

It should be noted also that we do not vary the gauge coupling parameter $g$ here. In the gauge $\mathcal{L}_{\xi} A_{\mu}=0$, it can be shown that $d\left(i_{\xi} A\right)$ is gauge invariant. Therefore $i_{\xi} A$ can only be shifted by a constant under the gauge transformation. We fix this residual gauge degree of freedom by requiring $\left.i_{\xi} A\right|_{\infty}=0$.

For our spherically-symmetric dyon, from the large $r$ expansion discussed in section 2 , we have

$$
\tilde{h}-h=\Delta_{1}+\frac{\Delta_{2}}{\rho}+\cdots, \quad \phi=\frac{\phi_{1}}{\rho}+\frac{\phi_{2}}{\rho^{2}}+\cdots,
$$

where $\rho$ is defined by (2.6) and

$$
\begin{aligned}
& \Delta_{1}=\frac{1}{4} g^{2} \phi_{1}^{2}, \quad \Delta_{2}=\frac{2}{3} g^{2} \phi_{1} \phi_{2}, \\
& \phi_{1}=-\frac{2 \sqrt{3} \mu\left(\beta_{1}-\beta_{2}\right)\left(1-\beta_{1} \beta_{2}\right)}{\gamma_{1} \gamma_{2}}, \quad \phi_{2}=\frac{2 \sqrt{3} \mu^{2}\left(\beta_{1}-\beta_{2}\right) \gamma}{\gamma_{1}^{2} \gamma_{2}^{2}} .
\end{aligned}
$$

Taking $\xi$ to be the time-like Killing vector $\xi=\partial / \partial t$, we find

$$
\delta \mathcal{H}=\int_{\infty}\left(\delta \mathcal{Q}_{\xi}-i_{\xi} \Theta\right)=\delta M+Z,
$$

where $M$ is the mass of the dyon given in (2.4) and $Z$ is given by

$$
Z=-\frac{1}{2} \delta \Delta_{2}+\frac{1}{2} g^{2} \phi_{2} \delta \phi_{1}+\frac{1}{4} g^{2} \phi_{1} \delta \phi_{2}=\frac{1}{12} g^{2}\left(2 \phi_{2} \delta \phi_{1}-\phi_{1} \delta \phi_{2}\right)
$$

Here we have set Newton's constant $G=1$. It is clear that $Z$ vanishes if $\phi_{2}=c \phi_{1}^{2}$, for any numerical constant $c$. Thus when $\beta_{1}=\beta_{2}$ and hence $\phi_{i}=0$, or when $\beta_{1}=0$ or $\beta_{2}=0$, for which $\phi_{2}=\mp \phi_{1}^{2} /(2 \sqrt{3})$, the quantity $Z$ vanishes and the usual thermodynamics holds. For generic $\beta_{i}$, we find

$$
Z=-X d Y
$$

where $(X, Y)$ are precisely the quantities introduced in section 2 in order to make the first law of thermodynamics to work. The procedure above shows that the quantities $(X, Y)$ depend only on the asymptotic parameters and are independent of properties at the horizon. 
The inclusion of a scalar charge in the thermodynamics was discussed in the context of asymptotically-flat black holes in [34. The details were rather different there, with the scalar charge being a modulus corresponding to the value $\phi_{\infty}$ of the scalar field at infinity, on account of the different asymptotic behaviour in that case. In contrast, our solution always has $\phi_{\infty}=0$, the stationary point of the scalar potential. A phenomenon that is more similar to the one we have encountered in this paper arose for asymptotically-AdS black holes in conformal gravity [29, 30, where the infinitesimal Hamiltonian is not integrable and the term $\int_{\infty} i_{\xi} \Theta$ leads to the inclusion of extra canonical pairs. In fact, the parallel to [30] is stronger, since in [30] extra canonical pairs beyond the total number of integration constants had to be introduced also.

In general the scalar $\phi$ approaches the asymptotic AdS as in (6.5), where $\phi_{1}$ and $\phi_{2}$ can in principle depend on other coordinates as well, but are constants for our static dyon. There are three types of boundary conditions that preserve all the asymptotic AdS symmetries. The first two are either $\phi_{1}=0$ or $\phi_{2}=0$ [35, 36]. The third is where $\phi_{2}=c \phi_{1}^{2}$ [37] (see also [38.) Interestingly, in all these three cases the quantity $Z$ vanishes. Thus, the quantity $Z$ we obtained via the Wald procedure describes scalar hair that breaks some of the asymptotic AdS symmetries.

\section{Conclusions}

In this paper, we constructed the static AdS dyonic black hole in four-dimensional maximal gauged supergravity, where one of the $U(1)$ gauge fields carries both electric and magnetic charges. If the gauge coupling constant is sent to zero, the solution reduces to the well-known asymptotically-flat Kaluza-Klein dyon. Our solution can be lifted to eleven dimensions, where it provides a new solution of M-theory. Since the four-dimensional solution is asymptotic to AdS, the gauged dyon provides a new background for exploring the AdS/CFT correspondence.

An intriguing feature arises when we study the thermodynamics of the gauged dyonic black hole. The naively-expected first law $d M=T d S+\Phi_{Q} d Q+\Phi_{P} d P$ does not hold except in the special cases enumerated in (1.4). In general, we find that we have to introduce a new pair of thermodynamic conjugate variables $(X, Y)$ in the first law, as in (2.13). By examining the asymptotic fall-offs and employing the Wald procedure for calculating the conserved quantities associated with the time-like Killing vector, we find that $X$ and $Y$ describe the potential and charge of the scalar hair that breaks some of the asymptotic 
AdS symmetries. This is the first explicit analytical example of a charged black hole with neutral scalar charge in gauged supergravity. It may be that a more general four-parameter class of static gauged dyonic black holes exists, where the scalar charge $Y$ can be specified independently of $M, Q$ and $P$, with our solution being a special case where $Y$ is a specific function of $(M, Q, P)$. If such a more general solution were known, it could shed further light on the physical meaning of the quantities $X$ and $Y$, and the possible interpretation of including an $X Y$ term in a Legendre transformation of the free energy. Since our AdS dyon is a solution to maximum gauged supergravity and is dual to an $\mathcal{N}=8, D=4$, superconformal field theory, it would be of great interest to study the gauge duals of the scalar hair.

\section{Acknowledgement}

We are grateful to Gary Gibbons and Andy Strominger for useful discussions. H.L. is supported in part by the NSFC grants 11175269 and 11235003. C.N.P. is supported in part by DOE grant DE-SC0010813.

\section{References}

[1] P. Dobiasch and D. Maison, Stationary, spherically symmetric solutions of Jordan's unified theory of gravity and electromagnetism, Gen. Rel. Grav. 14, 231 (1982).

[2] A. Chodos and S. L. Detweiler, Spherically symmetric solutions in five-dimensional general relativity, Gen. Rel. Grav. 14, 879 (1982).

[3] D. Pollard, Antigravity and classical solutions of five-dimensional Kaluza-Klein theory, J. Phys. A 16, 565 (1983).

[4] G.W. Gibbons and D.L. Wiltshire, Black holes in Kaluza-Klein theory, Annals Phys. 167, 201 (1986) [Erratum-ibid. 176, 393 (1987)].

[5] G.W. Gibbons and R.E. Kallosh, Topology, entropy and Witten index of dilaton black holes, Phys. Rev. D 51, 2839 (1995), hep-th/9407118.

[6] D. Rasheed, The rotating dyonic black holes of Kaluza-Klein theory, Nucl. Phys. B 454, 379 (1995), hep-th/9505038. 
[7] H. Lü, C.N. Pope and K.W. Xu, Liouville and Toda solutions of M theory, Mod. Phys. Lett. A 11, 1785 (1996), hep-th/9604058.

[8] H. Lü and W. Yang, SL(n,R)-Toda black holes, arXiv:1307.2305 [hep-th].

[9] K. Goldstein, N. Iizuka, R.P. Jena and S.P. Trivedi, Non-supersymmetric attractors, Phys. Rev. D 72, 124021 (2005), hep-th/0507096.

[10] R. Kallosh and B. Kol, E(7) symmetric area of the black hole horizon, Phys. Rev. D 53, 5344 (1996), hep-th/9602014.

[11] M. Cvetič and C. M. Hull, Black holes and U duality, Nucl. Phys. B 480, 296 (1996), hep-th/9606193.

[12] H. Liu, H. Lü and Z.-L. Wang, Killing spinors for the bosonic string and the KaluzaKlein theory with scalar potentials, Eur. Phys. J. C 72, 1853 (2012), arXiv:1106.4566 [hep-th].

[13] H.-S. Liu, H. Lü and Z. -L. Wang, Gauged Kaluza-Klein AdS pseudo-supergravity, Phys. Lett. B 703, 524 (2011), arXiv:1107.2659 [hep-th].

[14] K. Behrndt, M. Cvetič and W.A. Sabra, Nonextreme black holes of five-dimensional $\mathcal{N}=2$ AdS supergravity, Nucl. Phys. B 553, 317 (1999), hep-th/9810227.

[15] M.J. Duff and J.T. Liu, Anti-de Sitter black holes in gauged $\mathcal{N}=8$ supergravity, Nucl. Phys. B 554, 237 (1999), hep-th/9901149.

[16] M. Cvetič, M.J. Duff, P. Hoxha, J.T. Liu, H. Lü, J.X. Lu, R. Martinez-Acosta, C.N. Pope, H. Sati and T.A. Tran, Embedding AdS black holes in ten dimensions and eleven dimensions, Nucl. Phys. B 558, 96 (1999), hep-th/9903214.

[17] M. Cvetič, H. Lü and C.N. Pope, Gauged six-dimensional supergravity from massive type IIA, Phys. Rev. Lett. 83, 5226 (1999), hep-th/9906221.

[18] H. Lü, Charged dilatonic AdS black holes and magnetic $A d S_{D-2} \times R^{2}$ vacua, arXiv: 1306.2386[hep-th].

[19] A. Ashtekar and A. Magnon, Asymptotically anti-de Sitter space-times, Class Quant Grav 1, L39 (1984).

[20] A. Ashtekar and S. Das, Asymptotically anti-de Sitter space-times: Conserved quantities, Class. Quant. Grav. 17, L17 (2000), hep-th/9911230. 
[21] W. Chen, H. Lü and C.N. Pope, Mass of rotating black holes in gauged supergravities, Phys. Rev. D 73, 104036 (2006), hep-th/0510081.

[22] M. Cvetič, G.W. Gibbons, D. Kubiznak and C.N. Pope, Black hole enthalpy and an entropy inequality for the thermodynamic volume, Phys. Rev. D 84, 024037 (2011), arXiv:1012.2888 hep-th].

[23] S. de Haro, S.N. Solodukhin and K. Skenderis, Holographic reconstruction of space-time and renormalization in the AdS/CFT correspondence, Commun. Math. Phys. 217, 595 (2001), hep-th/0002230.

[24] J.D. Brown, E.A. Martinez and J.W. York, Jr., Complex Kerr-Newman geometry and black hole thermodynamics, Phys. Rev. Lett. 66, 2281 (1991).

[25] A. Chamblin, R. Emparan, C.V. Johnson and R.C. Myers, Charged AdS black holes and catastrophic holography, Phys. Rev. D 60, 064018 (1999), hep-th/9902170.

[26] M. Cvetič, H. Lü and C.N. Pope, Entropy-product rules for charged rotating black holes, arXiv:1306.4522[hep-th].

[27] M. Cvetič and D. Youm, Entropy of non-extreme charged rotating black holes in string theory, Phys. Rev. D54, 2612 (1996), hep-th/9603147.

[28] M. Cvetič, G.W. Gibbons and C.N. Pope, Universal area product formulae for rotating and charged black holes in four and higher dimensions, Phys. Rev. Lett. 106, 121301 (2011), arXiv:1011.0008[hep-th].

[29] H. Lü, Y. Pang, C.N. Pope and J.F. Vazquez-Poritz, AdS and Lifshitz black holes in conformal and Einstein-Weyl gravities, Phys. Rev. D 86, 044011 (2012), arXiv:1204.1062 [hep-th].

[30] H. Lü, Y. Pang and C.N. Pope, Black holes in six-dimensional conformal gravity, Phys. Rev. D 87, 104013 (2013), arXiv:1301.7083 [hep-th].

[31] R.J. Riegert, Birkhoff's theorem in conformal gravity, Phys. Rev. Lett. 53, 315 (1984).

[32] R.M. Wald, Black hole entropy is the Noether charge, Phys. Rev. D 48, 3427 (1993), gr-qc/9307038.

[33] V. Iyer and R.M. Wald, Some properties of Noether charge and a proposal for dynamical black hole entropy, Phys. Rev. D 50, 846 (1994), gr-qc/9403028. 
[34] G. W. Gibbons, R. Kallosh and B. Kol, Moduli, scalar charges, and the first law of black hole thermodynamics, Phys. Rev. Lett. 77, 4992 (1996), hep-th/9607108.

[35] P. Breitenlohner and D.Z. Freedman, Positive energy in anti-de Sitter backgrounds and gauged extended supergravity, Phys. Lett. B 115, 197 (1982).

[36] I.R. Klebanov and E. Witten, AdS/CFT correspondence and symmetry breaking, Nucl. Phys. B 556, 89 (1999), hep-th/9905104.

[37] T. Hertog and K. Maeda, Black holes with scalar hair and asymptotics in $\mathcal{N}=8$ supergravity, JHEP 0407, 051 (2004), hep-th/0404261.

[38] T. Hertog and G.T. Horowitz, Designer gravity and field theory effective potentials, Phys. Rev. Lett. 94, 221301 (2005), hep-th/0412169. 